\title{
The eminent need for an academic program in universities to teach nanomedicine
}

This article was published in the following Dove Press journal:

International Journal of Nanomedicine

22 August 201I

Number of times this article has been viewed

\section{Juan Manuel Vélez \\ Juan Jesus Vélez}

Intracellular Signaling Lab, Sección de Estudios de Posgrado e Investigación, Escuela Superior de Medicina, Instituto Politécnico Nacional, México
Correspondence: Juan Manuel Vélez Laboratorio de Señalización Intracelular, Sección de Estudios de Posgrado e Investigación, Escuela Superior de Medicina, Instituto Politécnico Nacional, México

Tel + I 5557296000 ext 62824

Email jvelez@ipn.mx
Abstract: Nanomedicine is on the cutting edge of technology applied to medical and biological sciences. Nanodevices, nanomaterials, nanoinstruments, nanotechnologies, and nanotechniques (laboratory methods and procedures) are important for the modern practice of medicine and essential for research that could stimulate the discovery of new medical advances. Accordingly, there is an eminent need for implementing an academic program in universities to teach this indispensable and pragmatic discipline, especially in the departments of graduate studies and research in the areas of pharmacology, genetic engineering, proteomics, and molecular and cellular biology.

Keywords: nanodevices, nanotechnologies, nanotechniques, nanobiosensors, quantum dots

\section{Introduction}

To be in step with the future, it is necessary today for academic programs to exist that teach topics related to nanomedicine, an important and relatively recent technological advance in medicine. Such programs should be included in university departments for graduate studies and research, especially in the areas of pharmacology, genetic engineering, proteomics, and molecular and cellular biology. Later, as nanotechnology continues to develop, other areas will certainly be affected.

Research in nanomedicine began with discoveries of the novel physical and chemical properties of various carbon-based materials that can only be detected in nanometer-sized structures. Nanomedicine is defined as the monitoring, repair, construction, and control of human biological systems at the cellular, molecular, and atomic levels using engineered nanodevices and nanostructures. Another definition is that provided by the National Institute of Health in its roadmap for medical research in nanomedicine, which states that nanomedicine is "an offshoot of nanotechnology, [which] refers to highly specific medical interventions at the molecular scale for curing disease or repairing damaged tissues, such as bone, muscle, or nerve". ${ }^{1}$ Nanomedicine contains five main disciplines: analytical tools, nanoimaging, nanomaterials (medications, raw materials, and devices), novel therapeutic and drug delivery systems, as well as regulatory and toxicological issues related to clinical practice. ${ }^{1}$

Basic nanostructured materials, engineered enzymes, and the many products of biotechnology will most probably become essential elements in some areas of medical, chemical, and biological applications. However, the full promise of nanomedicine is yet to be defined, as this will to a large extent depend on the development of new technology, such as precisely controlled or programmable medical nanomachines 
and nanorobots to address medical problems. Moreover, the application of nanotechniques at the cellular, molecular, and atomic levels should certainly help in the understanding of the function of organisms, as well as in the development of better techniques for imaging, diagnosing, and treating disorders at the level of molecular and cellular biology.

Microscopic machines were first hypothesized by the Nobel Prize winning physicist Richard P Feynman, now considered the father of nanoscience, in 1959. His article "There is Plenty of Room at the Bottom" is considered a classic in the establishment of the important possibilities for this field. ${ }^{2}$ The main idea of this article was the ability to reduce the size of various apparatuses and at the same time increase their effectiveness, based on the manufacture of products by reordering atoms and molecules. Of particular importance in his proposal was the reference to nanorobots, to be used in the manufacture of nanodevices, nanomaterials, and nanomachines. He also mentioned the possibility of creating a 100-fold improvement in the electron microscope.

Eric Drexler, considered the father of nanotechnology, wrote the popular books, Engines of Creation (1986) and Unbounding the future (1991), in which he announced the promise and warned of the dangers of molecular and atomic manipulation. In his more recent work, Nanosystems: molecular machinery, manufacturing and, computation (1992), he predicted that nanotechnology will make the conventional means of manufacture obsolete. ${ }^{3}$ He proposed the concept of machines with the capability of ordering and reordering atoms in order to create precise molecular structures, thus resulting in the desired product. Such machines could even have the ability of self-reproduction.

Once nanomachines are available, programmable and controllable microscale robots comprised of nanoscale parts manufactured to nanometer precision will open new possibilities related to curative and reconstructive procedures in the human body at the cellular, molecular, and atomic levels (perhaps at less than $100 \mathrm{~nm}$ ). In keeping with the adage that "the best treatments are those that intervene the least", the possibility of nanotechnology to greatly increase knowledge of cellular and atomic events could lead to improved treatments. Additionally, nanomedicine is on the cutting edge of medical practice and research, and for this reason it is an important focus of capital investment. Consequently, it is urgent that academic programs be implemented in order to teach this discipline in universities that have a medical and biological focus of study and research.

An academic discipline of nanomedicine at the university level would allow students, teachers, technicians, and researchers to become familiar with: (1) nanodevices, nanostructures, nanomaterials, nanostuffs, and precise and sophisticated nanoinstruments, such as those that could be used for molecular imaging and bedside biomarker testing and monitoring (Appendix 1); (2) the main categories of precise nanotechnologies in real time (Appendix 2); and (3) techniques (laboratory methods and procedures) needed for furthering research (Appendix 3), including detailed procedures of nanobiosensors for medical uses (Appendix 4). All of these elements of nanomedicine could be applied to cancer, cardiovascular diseases, inflammation, degenerative neurological diseases, toxicity, ethics, and societal and environmental impact. ${ }^{4}$

Perhaps one of the most important potentials for nanomedical research is the gathering of extensive information about the chemical and physical properties of nanoscale biological structures. As the catalog of the interactions between individual molecules, cells, tissues, and organs develops, researchers will gain a greater understanding of the intricate operations of molecular structures, processes, and networks inside living cells based on the use of new probing tools. Consequently, scientists will be able to build new devices for a wide range of biomedical applications, such as detecting infectious agents or metabolic imbalances with tiny sensors, generating miniature devices that search for and destroy infectious agents, and replacing "broken" machinery inside cells with new nanoscale structures. ${ }^{4}$

\section{Current applications}

The following medical advances are among the existing uses of nanomedicine.

- Nanomaterials: nanotechnology used for subcutaneous treatments. $^{5}$

- Nanoprobes in vivo: targeted nanoprobes reveal early time point kinetics in vivo by time-resolved magnetic resonance imaging (MRI). ${ }^{6}$

- Methods for the preparation of small interfering ribonucleic acids (siRNAs): a direct, efficient method for the preparation of siRNAs containing ribo-like north pseudo-sugars, ${ }^{7}$ and drug delivery of siRNA therapeutics. ${ }^{8}$

- Physical assessment of toxicology at nanoscale: nanodose-metrics and toxicity factor. ${ }^{9}$

- Nanomaterials for regenerative medicine: use of nanomaterials with applications for regenerative medicine. ${ }^{10}$

- Vascular toxicity: studies of vascular endothelial toxicity. ${ }^{11}$

- Nanocatalizers: molecular-level biological transporters capable of converting biomass into biofuel. 
- Nanoparticle devices: molecular-level biological transporters that release medication in a precise and controlled manner throughout the organism, such as in target cells, for crossing biological barriers and for providing greater stability in the face of pathogenic deterioration, as well as use of nanoparticles for the treatment of cancer. ${ }^{12}$

\section{Future directions and challenges}

New opportunities will most certainly appear in relation to fighting disease as nanotechnology develops. Doctors may be able to search out and destroy the very first cancer cells before they lead to a destructive tumor in the body. Perhaps a broken part of a cell could be removed and replaced with a miniature biological machine. Pumps the size of molecules could possibly be implanted to deliver life-saving medicines precisely when and where they are needed. These scenarios are the long-term goals of the "nanomedicine roadmap". ${ }^{4}$ With the inclusion of the academic discipline of nanomedicine in their programs, universities will be able to provide the personnel capable of accelerating the development of this field. Future possibilities include the following opportunities:

- Nanoneedles: the nucleus of live cells could be penetrated by nanoneedles used in conjunction with microscopy with the aim of introducing nucleic acids, proteins or other compounds inside human cells, or even doing surgery; modeling the structure-property relationships of nanoneedles. ${ }^{13}$

- Environmental nanocatalizers: biological transporters capable of greatly improving catalytic converters currently used in automobiles, thus drastically reducing the pollution emitted.

- Nanobiosensors: sensors capable of detecting the presence of anthrax and other biological weapons; nanosensors for probing live cells. ${ }^{14}$

- Dendrimers: artificial molecules designed to replace defective endogenous elements in the organism, induce the production or secretion of various molecules related to the immune response in case of being genetically deficient, or act as biological markers (for cancer therapy, for the detection of adherence to medication regimens, or for the evaluation of experimental results). ${ }^{15}$

- Nanodepots of insulin: microscopic deposits injected into the bloodstream that could act as an artificial pancreas, releasing insulin to counteract the effects of diabetes; similar technologies could also be employed to release dopamine into neurons as therapies for Parkinson's or Alzheimer's disease.

- Titanium dioxide nanotubes: microscopic tubes that could clean polluted water.
- Carbon nanotubes: microscopic tubes aimed at replacing defective or absent genes; use of carbon nanotubes in vivo to characterize action mechanisms at the cellular level; ${ }^{16}$ carbon nanotubes could reactivate synaptic connections and regenerate neurons damaged by an accident.

- Biological nanosensors: a set of ultramicroscopic and fine silicon threads.

- Molecular nanopyramids: these probes could possibly be released into the human body, with the capability of detecting and destroying the first cancer cells in the brain, heart, kidney, or lungs, without damaging neighboring tissue; they might also be able to detect viral or bacterial infections in an early stage before the immune system has begun to respond.

- Assembler nanomachines: machines based on the rearrangement of atoms and molecules to manufacture molecular components.

- Smart materials: these probe-like materials could potentially detect subtle changes in body chemistry.

- Nanoparticles: microscopic tumor-destroying genes may possibly be transported to certain sites, directed with great precision to destroy the genetic information of tumors, with the aim of inactivating the target cells and impeding their reproduction; other genes may be transported to detect tiny alterations in proteins, as well as to scan tissue to ensure that the nanodevices are neatly congregated around the target.

- Nanorobots: these devices could be introduced into the human body to repair damage and combat infection and disease by acting on DNA without being detected and destroyed by the host immune system. ${ }^{17-22}$

- Nanobiochips: this advanced technology could work with MRI to supply an abundant amount of information about the human body. ${ }^{23}$

- Nanocomputers: computers could be developed made of material stronger than steel but with only $10 \%$ of its weight. These could possibly guide nanomachines for the purpose of examining, taking apart, and rebuilding damaged cells, tissues, and organs through the reconstruction of their molecular structures; nanoinformatics could be applied to nanomedicine; ${ }^{24}$ new computers could search for a methodology that reproduces human organs in vitro.

\section{Conclusion}

The academic discipline of nanomedicine could be included in university curriculums for graduate students and researchers today in the fields of pharmacology, genetic engineering, proteomics, and molecular and cellular biology. Such programs 
would train personnel in order to accelerate advances of techniques, technology, and applications of nanomedicine. As such uses increase and broaden, other new programs will also become important elements of university curriculums.

\section{Acknowledgments}

I thank AY Vélez and MPE Arvízu for help in the use of the English language. Thanks to AS Vélez for his help in describing the use of different apparatus employed in nanotechnology.

\section{Disclosure}

The authors report no conflicts of interest in this work.

\section{References}

1. National Institutes of Health (NIH). National Institutes of Health Roadmap for Medical Research: Nanomedicine. 2006. Available from: http:// nihroadmap.nih.gov/nanomedicine. Accessed June 5, 2011.

2. Feynman RP. There's plenty of room at the bottom. Caltech Engineering and Science. 1960;23(5):22-36.

3. Drexler E. Nanosystems: Molecular Machinery Manufacturing, and Computation. Nanotechnology, Chapter 4. Hoboken, NJ: John Wiley; 1992.

4. Webster, Thomas J. Nanomedicine: what's in a definition? Int $J$ Nanomedicine. 2006;1(2):115-116.

5. Demming A. Nanotechnology under the skin. Nanotechnology. 2011; 22(26):260201.

6. Bhirde A, Guo N, Chen X. Targeted nanoprobes reveal early time point kinetics in vivo by time-resolved MRI. Theranostics. 2011;1:274-276.

7. Terrazas M, Aviñó A, Siddiqui MA, Marquez VE, Eritja R. A direct, efficient method for the preparation of siRNAs containing ribo-like north bicyclo[3.1.0]hexane pseudosugars. Org Lett. 2011;13(11): 2888-2891.

8. Reischl D, Zimmer A. Drug delivery of siRNA therapeutics: potentials and limits of nanosystems. Nanomedicine. 2009;5(1):8-20.
9. Pompa PP, Vecchio G, Galeana A, et al. Physical assessment of toxicology at nanoscale: nano dose-metrics and toxicity factor. Nanoscale. May 6, 2011. [Epub ahead of print].

10. Verma S, Domb AJ, Kumar N. Nanomaterials for regenerative medicine. Nanomedicine (Lond). 2011;6(1):157-181.

11. Yan M, Zhang Y, Xu K, Fu T, Qin H, Zheng X. Vascular toxicity: an in vitro study of vascular endothelial toxicity of CdTe quantum dots. Toxicology. 2011;282(3):94-103.

12. Aggarwal S, Yadav S, Gupta S. EGFR targeted PLGA nanoparticles using gemcitabine for treatment of pancreatic cancer. $J$ Biomed Nanotechnol. 2011;7(1):137-138.

13. Poater A, Saliner AG, Carbó-Dorca R, et al. Modeling the structureproperty relationships of nanoneedles: a journey toward nanomedicine. J Comput Chem. 2009;30(2):275-284.

14. Kneipp J, Kneipp H, Wittig B, Kneipp K. Novel optical nanosensors for probing and imaging live cells. Nanomedicine. 2010;6(2): 214-226.

15. Cheng Y, Zhao L, Li Y, Xu T. Design of biocompatible dendrimers for cancer diagnosis and therapy. Chem Soc Rev. 2011;40(5):2673-2703.

16. Al Faraj A, Fauvelle F, Luciani N, et al. Use of carbón nanotubes: in vivo biodistribution and biological impact of injected carbón nanotubes using magnetic resonance techniques. Int J Nanomed. 2011; 6:351-361.

17. Freitas RA Jr. Current status of nanomedicine and medical nanorobotics. J Comput Theor Nanosci. 2005;2:1-25.

18. Kostarelos K. Narobots for medicine. Nanomedicine (Lond). 2010;5 (3):341-342.

19. Maojo V, Martin-Sanchez F, Kulikowski C, Rodriguez-Paton A, Fritts M. Nanoinformatics and DNA-based computing: catalyzing nanomedicine. Pediatr Res. 2010;67(5):481-489.

20. Hoggt T. Maintaining your health from within. Controls for nanorobots swarms in fluids. Stud Health Technol Inform. 2009;149:311-321.

21. Patel GM, Patel GC, Patel RB, Patel JK, Patel M. Nanorobot: a versatile tool in nanomedicine. J Drug Target. 2006;14(2):63-67.

22. Cavalcanti A, Freitas RA Jr. Nanorobotics control design: a collective behavior approach for medicine. IEEE Trans Nanobioscience. 2005;4(2):133-140.

23. Jung M, Oh BK, Choi JW. Fabrication of Au nanodots with $60 \mathrm{~nm}$ diameter on ITO glass: towards nanobiochips using nanoporus alumina mask. Ultramicroscopy. 2009;109(8):1006-1010.

24. De la Iglesia D, Maojo V, Chiesa S, et al. Nanoinformatics research applied to nanomedicine. Method Inf Med. 2011;50(1):84-95. 


\section{Appendices}

Appendix I Nanomaterials, nanodevices, nanoinstruments, and nanostuffs useful in nanomedicine

\begin{tabular}{llll}
\hline Nanostructures & Nanoparticles & Nanofibers & Nanoplatforms \\
Nanodisks & Nanoscaffolds & Nanolabels & Nanodiamonds \\
Nanoarrays & Nanocomposites & Nanopackages & Nanobubbles \\
Nanobeads & Nanofluidics & Nanobiosensors & Nanogadgets \\
Nanofilaments (silicone) & Nanotargets & Nanofilms & Nanoscale \\
Nanotubes (carbon) & Nanorods & Nanoshapes & Nanocoatings \\
Nanosuspensions & Nanoemulsions & Nanocarriers & Nanospheres \\
Nanosystems & Nanogels & Nanocontainers & Nanoproducts \\
Nanodrugs & Nanovectors & Nanohybrids & Nanoreactives \\
Nanoscopic & Nanoclusters & Nanobullets & Nanoknittings \\
Nanoswitches & Nanoblobs & Nanohazards & Nanobodies \\
Nanosurfaces & Nanopores & Nanocatalysts & Nanotools \\
Nanochips & Nanorobots & Nanocrystals & Nanoneedles \\
Nanowires (silicone) & Nanoshells & Nanoencapsulation & Nanoprobes \\
Nanolayers & Nanomotors & Nanomachines & Nanocages \\
Nanoimpellers & Nanomatrix & Pharmacytes & Dendrimers \\
Nanoscopic weapons & Qubit & SPION & Quantum dots \\
\hline Abbrevion & SPNON &
\end{tabular}

Abbreviation: SPION, superparamagnetic iron oxide nanoparticles.

\section{Appendix 2 Technologies}

MIDD: molecular imaging drug delivery Nanoparticle drug delivery systems

Tissue engineering regenerative medicine Cell therapy regenerative medicine UMI: ultrasound molecular imaging Wireless endoscopy and biotelemetry AFM: atomic force microscopy Nanoparticles for molecular diagnostics Stem cell repair and therapy Stem cell therapy for cancer Nanoparticles for tracking stem cells

Nanoscale single-cell or molecule identification

Nanotechnology-based cytogenetics

Cancer molecular imaging

Nanotechnology-based biochips and microarrays

Virtual reality stimulation

Phototermical therapy

Biodegradable polymeric nanoparticles as drug delivery devic

Modular robotics, multi-agent or swarm robotics, and utility fog

Appendix 3 Techniques and methods

QDs broad absorption spectra

FISH: florescent in situ hybridization

Atomic force microscopy and

quantum dot

Scanning near-field optical microscopy

Functional polymer-coated nanoparticles

Lumininescent fluorescence microscopy

Immunicon's cell tracks technology

Artificial engineering

Nanoscopic medicine

Nanodimensional three

Cell surface engineering

Nanodiagnostics

Regenerative medicine

Nanofluidic technology

Stem cell tissue engineering

Nanocybernetics

Laser nanoscissor

SPR: surface plasmon

resonance

Molecular diagnostics

Endothelial cell repair

Nanoscale probes

RAMiTS technology

Nanoproteomics

Organic electronic 


\section{Appendix 4 Nanobiosensors}

\begin{tabular}{ll}
\hline Nanowire biosensors & $\begin{array}{l}\text { lon channel switch biosensor } \\
\text { technology }\end{array}$ \\
Cantilevers as biosensors & $\begin{array}{l}\text { Electrochemical nanobiosensors } \\
\text { Carbon nanotube biosensors }\end{array}$ \\
Electronic nanobiosensors & Quartz nanobalance DNA sensor \\
Viral nanosensor & Nanosensors based on surface \\
& plasmon resonance \\
Optical biosensors using laser, & PEBBLE \\
nanoshell, SPR, SERS, mRNA &
\end{tabular}

Abbreviations: FRET, fluorescence resonance energy transfer; PEBBLE, probes encapsulated by biologically localized embedding; SERS, surface enhanced Raman scattering; SPR, surface plasmon resonance.

\section{Publish your work in this journal}

The International Journal of Nanomedicine is an international, peerreviewed journal focusing on the application of nanotechnology in diagnostics, therapeutics, and drug delivery systems throughout the biomedical field. This journal is indexed on PubMed Central, MedLine, CAS, SciSearch ${ }^{\circledR}$, Current Contents ${ }^{\circledR} /$ Clinical Medicine,
Journal Citation Reports/Science Edition, EMBase, Scopus and the Elsevier Bibliographic databases. The manuscript management system is completely online and includes a very quick and fair peer-review system, which is all easy to use. Visit http://www.dovepress.com/ testimonials.php to read real quotes from published authors.

\footnotetext{
Submit your manuscript here: http://www.dovepress.com/international-journal-of-nanomedicine-journal
} 